\title{
GAS TEMPERATURE DISTRIBUTION AND EDDY DIFFUSIVITY OF HEAT IN GASEOUS SUSPENSION FLOW
}

\author{
SHIGERU MATSUMOTO, AKIHIKo TAKAHASHI, \\ MUTSUMI SUZUKI AND SiRo MAEDA \\ Department of Chemical Engineering, \\ Tohoku University, Sendai 980
}

\begin{abstract}
Two mathematical models are proposed to represent gas temperature distributions in suspension flows heated at the tube wall. These models are quite different from each other in the turbulent mixing mode of solid particles. Two parameters in these models were determined from experimental measurement of temperature distribution by using a parameter estimation technique. These models express observed temperature distributions well, especially when the loading ratio is smaller than unity. Mean gas eddy diffusivity and Nusselt number were calculated from these two parameters, which could be correlated well with a single nondimensional parameter that represents the operating conditions. By using this correlation and the models, temperature distributions can be easily predicted in the usual practical operating conditions of industrial suspension flows.
\end{abstract}

\section{Introduction}

In recent years, a number of studies have been made of gaseous suspension flow accompanying heat exchange with a boundary, because of its wide application in industry. Most of them have shown greatest interest in the heat transfer coefficient at the boundary surface. A considerable amount of experimental data for the heat transfer coefficient at the tube wall are now available, and effects of various parameters such as mass flow ratio, particle size and Reynolds number have become clearer in outline. In a previous paper ${ }^{6}$, we presented a general correlation of wall-heat transfer coefficients in vertical gas-solid suspension flows for uniform wall temperature conditions.

In designing equipment for many industrial operations which involve a gaseous suspension flow system, such as transfer line, synthesis reactor and catalytic cracking units, chemical engineers have to consider the temperature distribution of gas in a reactor and the solid temperature within a duct as well as the wallheat transfer coefficient. Unfortunately, only a little information has been available about these aspects except for the experiments in horizontal flow by Briller and Peskin ${ }^{4}$.

There has also been only a little work on the theoretical analysis of this problem. Tien ${ }^{8\rangle}$ made the first

Received September 2, 1978. Correspondence concerning this article should be addressed to M. Suzuki. A. Takahashi is now with Mitsui Toatsu Chemicals Co., Ltd., Nagoya 457. analytical attempt to predict the effect of particulate matter on the heat transfer coefficient, where the problem became a Strum-Liouville eigen value problem. This analysis was extended by Depew and Farbar ${ }^{5}$, and Boothroyd ${ }^{3}$. But these analyses are limited to cases of fine suspensions, and require troublesome calculations.

In this paper, simple mathematical models are presented for the heat transfer mechanism in a vertical suspension flow, and consideration is given to the temperature distribution of gas phase, heat transfer coefficient at wall and gas eddy diffusivity of heat, compared with experiments previously presented. Further, we are concerned with an estimation method for gas temperature distribution and wall heat transfer coefficient in any gaseous suspension flow.

\section{Theoretical Analysis}

To develop a theoretical expression of this system, we need some information about the heat transfer characteristics of solids as well as the flow characteristics of phases. However, there has been little work on the former, most of it concerned with solid eddy diffusivity of heat. In this paper we will present two typical models in respect to solid eddy diffusivity of heat in the radial direction of the tube. First, in Model I, eddy diffusivity is neglected. This implies no eddy-mixing of solid particles in the radial direction within a tube. On the other hand, in Model II, the mixing of solid particles is so violent that eddy 
diffusivity of heat is extremely large, resulting in a uniform solids temperature distribution over the crosssection of the tube.

The basic assumptions required for the system are as follows:

1) Fluid and solid properties are constant and uniform.

2) The two-phase flow system is fully developed at the heat transfer section inlet.

3) Velocity profiles of phases are uniform over the pipe cross-section.

4) Axial heat transfer due to turbulent diffusion is negligible compared with that due to convection.

5) At the tube wall, heat is transferred only to the gas phase.

6) Radiation effects are neglected.

7) The particle Biot number is sufficiently small and the temperature is uniform within the particle.

\section{1 Model I (No-solid mixing model)}

In addition to the above fundamental assumptions, this model is restricted by the following assumptions. a) The gas eddy diffusivity of heat is uniform over the pipe cross-section except in the region of the wall vicinity. The extremely small eddy diffusivity in the vicinity of the wall is described as a form of film coefficient.

b) The solid concentration is uniformly distributed throughout the pipe cross-section.

c) Solid eddy diffusivity of heat is neglected; that is, mixing of solids due to turbulent diffusion never occurs in the radial direction.

Then the heat balance equations for phases are derived as

$$
\begin{aligned}
& \frac{\partial t_{g}}{\partial x}=\left(\frac{\varepsilon_{H}}{u_{g}}\right)\left(\frac{\partial^{2} t_{g}}{\partial r^{2}}+\frac{1}{r} \frac{\partial t_{g}}{\partial r}\right)-\left(\frac{n_{i} A_{p} h_{p}}{u_{g} \rho_{g} C_{p, g}}\right)\left(t_{g}-t_{s}\right) \\
& \frac{\partial t_{s}}{\partial x}=\left(\frac{n_{i} A_{p} h_{p}}{u_{s} \rho_{s} C_{p, s}}\right)\left(t_{g}-t_{s}\right)
\end{aligned}
$$

The boundary conditions for $t_{g}$ and $t_{s}$ are as follows:

$$
\begin{aligned}
& x=0 ; t_{g}=t_{g, 0}, t_{s}=t_{s, 0}\left(0 \leq r \leq r_{0}\right) \\
& x>0 ; \text { at } r=r_{0},-\rho_{g} C_{p, g} \varepsilon_{H}\left(\partial t_{g} / \partial r\right)+h_{B}\left(t_{w}-t_{g}\right)=0
\end{aligned}
$$

Equation (4) expresses assumption (a), where the very small eddy diffusion in the vicinity of the wall is described as the film coefficient $h_{B}$. With the dimensionless variables

$$
T_{g}=\begin{gathered}
t_{w}-t_{g} \\
t_{w}-t_{g, 0}
\end{gathered}, \quad T_{s}=\begin{gathered}
t_{w-t_{s}} \\
t_{w}-t_{g, 0}
\end{gathered}, R=\frac{r}{r_{0}}, X=\frac{x}{D}
$$

Eqs. (1)-(4) become

$$
\begin{aligned}
& \frac{\partial T_{g}}{\partial X}=\alpha\left(\frac{\partial^{2} T_{g}}{\partial R^{2}}+\frac{1}{R} \frac{\partial T_{g}}{\partial R}\right)-\beta\left(T_{g}-T_{s}\right) \\
& \frac{\partial T_{s}}{\partial X}=\gamma\left(T_{g}-T_{s}\right)
\end{aligned}
$$

$$
\text { B. C. } \left.\begin{array}{l}
X=0 ; T_{g}=1, T_{s}=T_{s, 0}(0 \leq R \leq 1) \\
X>0 ; \text { at } R=1, \frac{\partial T_{g}}{\partial R}+B T_{s}=0
\end{array}\right\}
$$

where

$$
\left.\begin{array}{l}
\alpha=4\left(\varepsilon_{H} / \nu\right) / R e=4 / P e \\
\beta=\left(n_{i} A_{p} h_{p} D\right) /\left(u_{g} \rho_{g} C_{p, g}\right)=m r\left(C_{p, s} / C_{p, g}\right) \\
\gamma=\left(n_{i} A_{p} h_{p} D\right) /\left(u_{s} \rho_{s} C_{p, s}\right)=\tau_{p} / \tau_{h p} \\
B=\left(h_{B} r_{0}\right) /\left(\rho_{g} C_{p, g} \varepsilon_{H}\right)=N u_{B} /(2 P r)\left(\varepsilon_{H} / \nu\right)
\end{array}\right\}
$$

Four dimensionless parameters appearing in the model are defined by Eq. (9). $\quad \alpha$ is a term relating to the gas eddy diffusivity and the reciprocal of the Peclet number, $\beta$ the heat capacity ratio of solids to gas, $\gamma$ the characteristic time ratio of motion to heat transfer rate of particle, and $B$ the film coefficient at the wall. Among them, $\beta$ and $\gamma$ can be evaluated from operating variables such as physical properties of solid material, mass flow ratio, gas velocity, conduit size, etc.*, but $\alpha$ and $B$ remain unknown owing to a lack of information about the gas eddy diffusivity in a suspension flow.

Applying the Laplace transform after the finiteHankel transform, analytical solutions of the above differential equations are obtained as

$$
\begin{aligned}
T_{g}= & 2 \sum_{i=1}^{\infty} \frac{B J_{0}\left(R \xi_{i}\right)}{\left(B^{2}+\xi_{i}^{2}\right) J_{0}\left(\xi_{i}\right)} \\
& \times \exp \left(-a_{1} X\right)\left[\cosh \left(b_{1} X\right)+\frac{c_{1}}{b_{1}} \sinh \left(b_{1} X\right)\right] \\
T_{s}= & 2 \sum_{i=1}^{\infty} \frac{B J_{0}\left(R \xi_{i}\right)}{\left(B^{2}+\xi_{i}^{2}\right) J_{0}\left(\xi_{i}\right)} \\
& \times \exp \left(-a_{1} X\right)\left[\cosh \left(b_{1} X\right)+\frac{c_{2}}{b_{1}} \sinh \left(b_{1} X\right)\right]
\end{aligned}
$$

where eigen values $\xi_{i}$ are positive roots of

$$
\xi_{i} J_{1}\left(\xi_{i}\right)=B J_{0}\left(\xi_{i}\right)
$$

and auxiliary variables are

$$
\begin{aligned}
& a_{1}=(1 / 2)\left(\alpha \xi_{i}^{2}+\beta+\gamma\right) \\
& b_{1}=\left(a_{1}^{2}-\alpha \gamma \xi_{i}^{2}\right)^{1 / 2} \\
& c_{1}=\beta T_{s, 0}+\gamma-a_{1} \\
& c_{2}=\left(\gamma / T_{s, 0}\right)-\gamma+a_{1}
\end{aligned}
$$

The procedures are presented briefly in the Appendix. Dimensionless mixed-mean temperatures of the phase at any distance from the heat exchanger inlet are respectively

$$
\begin{aligned}
& T_{g, \text { mean }}=2 \int_{0}^{1} R T_{g} d R \\
& =4 \sum_{i=1}^{\infty} \frac{B^{2}}{\left(B^{2}+\xi_{i}^{2}\right) \xi_{i}^{2}} e^{-a_{1} x}\left[\cosh \left(b_{1} X\right)+\frac{c_{1}}{b_{1}} \sinh \left(b_{1} X\right)\right]
\end{aligned}
$$

$$
T_{s, \text { mean }}=2 \int_{0}^{1} R T_{s} d R
$$

* Solid velocity $u_{s}$ is calculated from the gas velocity and the terminal velocity of a single particle. 


$$
=4 \sum_{i=1}^{\infty} \frac{B^{2}}{\left(B^{2}+\xi_{i}^{2}\right) \xi_{i}^{2}} e^{-a_{1} x}\left[\cosh \left(b_{1} X\right)+\frac{c_{2}}{b_{1}} \sinh \left(b_{1} X\right)\right]
$$

And the average rate of heat transfer at the wall over the pipe length $L$ is

$$
\begin{aligned}
Q_{\text {a》 }}= & \sum_{i=1}^{\infty} \frac{B^{2}}{\left(B^{2}+\xi_{i}^{2}\right)}\left[\begin{array}{c}
1+\left(c_{1} / b_{1}\right) \\
a_{1}-b_{1}
\end{array}\left\{1-e^{-\left(a_{1}-b_{1}\right)(L / D)}\right\}\right. \\
& \left.+\frac{1-\left(c_{1} / b_{1}\right)}{a_{1}+b_{1}}\left\{1-e^{-\left(a_{1}+b_{1}\right)(L / D)}\right\}\right]
\end{aligned}
$$

Then the overall Nusselt number based on the logarithmic mean temperature difference is

$$
N u_{m}=2 P r \cdot\left(\varepsilon_{H} / \nu\right) \cdot Q_{\mathrm{av}} /(\Delta T)_{m}
$$

where $(\Delta T)_{m}$ is a dimensionless logarithmic mean of terminal temperature difference between wall and mixed-mean gas temperature, as

$$
(\Delta T)_{m}=\left(T_{g, \text { mean }}-1\right) / \ln T_{g, \text { mean }}
$$

\section{2 Model II (Perfect solid mixing model)}

This is the opposite of Model I in respect to the eddymixing of solids in the radial direction; that is, the solid eddy diffusivity of heat is assumed to be extremely large, so that the temperature distribution of solids should be uniform throughout the tube crosssection. The other assumptions are the same as in Model I.

Thus, the basic equation for solid phase, Eq. (2), is rewritten in dimensionless form as

$$
\frac{d T_{s}}{d X}=2 \gamma \int_{0}^{1}\left(T_{g}-T_{s}\right) R d R=2 \gamma \int_{0}^{1} R T_{g} d R-\gamma T_{s, \text { mea n }}
$$

and the other equation and boundary conditions are the same as in Model I. However, this system is so difficult to solve analytically that we evaluated the numerical solutions by the Crank-Nicolson implicit method, where mesh size was $20 \times 200$.

\section{Experiment}

The experimental apparatus and the solids used in the present work are described in detail in a previous paper $^{6)}$. In brief, the apparatus is a vertical pressuretype transport system. The test section consists of a double-jacket heat exchanger, the inner tube of which is a $26-\mathrm{mm}$ I.D., $2 \mathrm{~m}$-long copper tube, and its wall temperature is maintained uniform at approximately $100^{\circ} \mathrm{C}$ by saturated steam. Gas temperature distributions in the tube were measured at the inlet and outlet of the test section by a thermocouple traversing probe. The heat transfer rate was determined by measuring inner-jacket condensate. The wall heat transfer coefficient was defined based on the logarithmic mean terminal temperature differences between wall and mixed-mean gas temperature.

\section{Results and Discussion}

\section{1 Comparisons between calculated and observed gas}

\section{temperature distributions}

In the above models, there are two unknown parameters, the gas eddy diffusivity ratio $\varepsilon_{H} / \nu$ in $\alpha$, and the wall Nusselt number $N u_{B}$ in $B$. Temperature distributions of gas at the test section outlet were calculated by varying those parameters, $\alpha$ and $B$, so as to fit the observations as well as possible.

Figures 1 and 2 respectively illustrate the comparisons of calculated results with experiments for $181 \mu$ and $1130 \mu$ glass beads at $R e=15,000^{*}$. The solid lines show the distributions calculated by Model I and the dotted lines by Model II. For $181 \mu$ beads, Model I agrees with experiment within the range of small loading ratios, to $m=2$ or so. But this model shows poor agreement for higher loading ratios because of core flattening of the gas temperature distributions. A similar tendency is seen in the case of $R e=24,000$, though the figures are abbreviated. For $1130 \mu$ beads shown in Fig. 2, no core flattening of distribution appears over this experimental range, so observed temperature distributions are expressed well by Model I.

On the other hand, Model II explains well the cases of both 181 and $1130 \mu$ beads, as shown in Figs. 1 and 2. However, even if the temperature distributions are explained by the models, the overall Nusselt numbers, $N u_{m}$, calculated by Eq. (17), do not agree exactly with experiment for either model, as shown in tables in the figures.

For the case of smaller particle size, $72 \mu$ glass beads shown in Fig. 3, temperature distributions by Model II show poor agreement with observed ones. This is caused mainly by the fact that solids concentrated remarkably in the central part of the pipe at higher loading ratios, as mentioned in the previous paper. In particular, for higher loading ratios gas temperature approaches solid temperature because the solid absorbs a lot of heat from gas phase due to its large surface area. In such a case, the temperature profile of solid is greatly reflected in that of gas. Consequently, the temperature profile of gas by Model II becomes flat in the core region. It seems, however, that Model I can express the experiments within relatively low loading ratios, less than 1 or so. Results for $321 \mu$ copper beads are presented in Fig 4 , where the overall Nusselt numbers as well as temperature distributions show good agreement with both the models. The reason is considered to be that gas temperature is not affected so much by solid addition because of the small value of $\beta$ due to high solid density.

\footnotetext{
* Comparisons were carried out at a distance of $X=769$. from the inlet of the heated section, where the value of the dimensionless parameter, $2 x / D R e P r$, ranged from $0.956 \times 10^{-2}$ to $1.83 \times 10^{-2}$. The distance might be long enough to cover the thermal entrance length.
} 


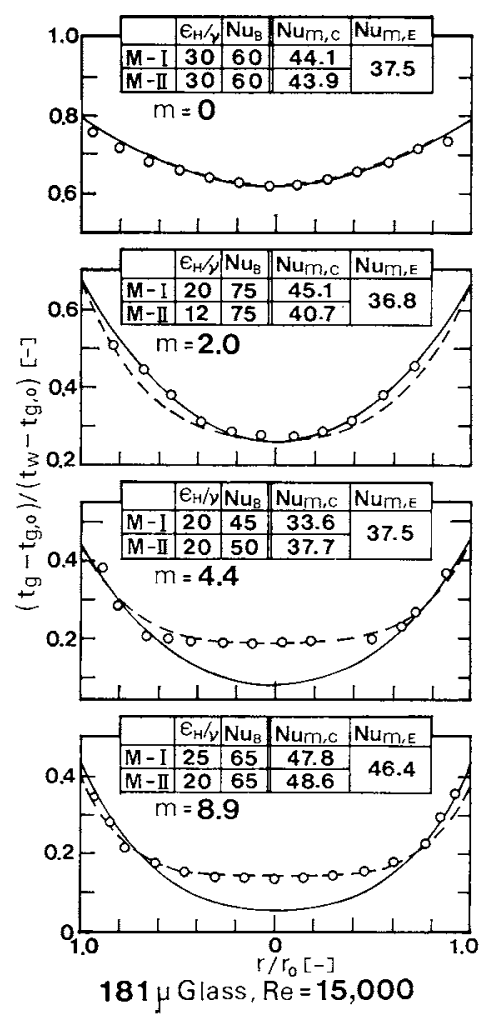

(Solid lines indicate Model I and dotted lines Model II.)

Fig. 1 Comparison of calculated gas temperature distributions with observed ones

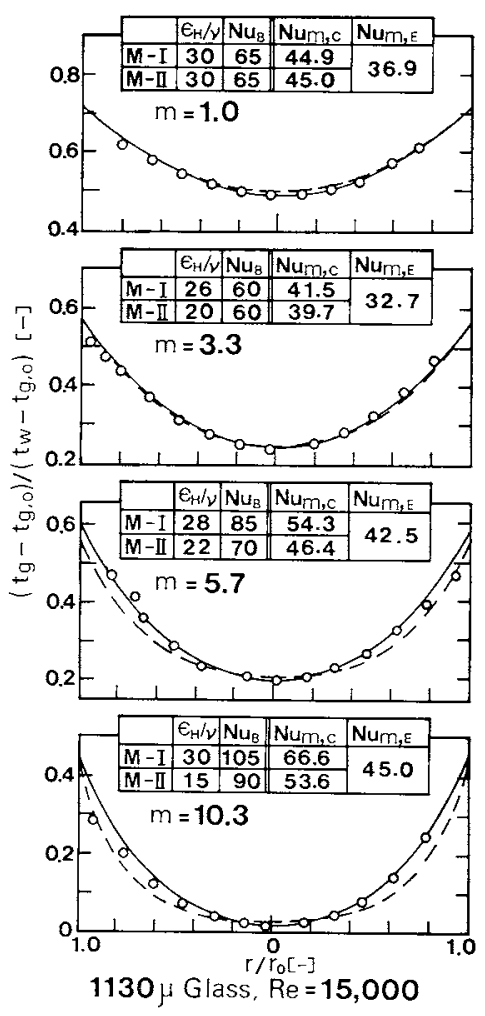

Fig. 2 Comparison of calculated gas temperature distributions with observed ones

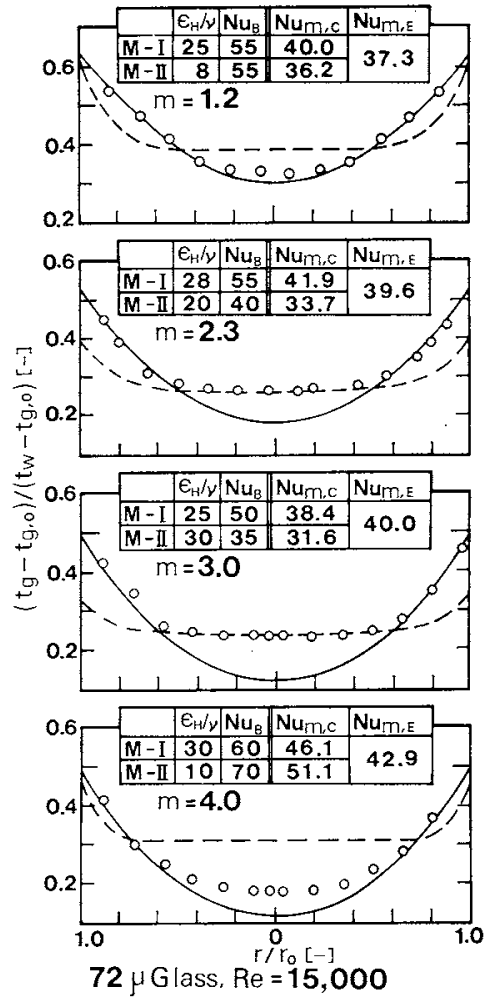

Fig. 3 Comparison of calculated gas temperature distributions with observed ones

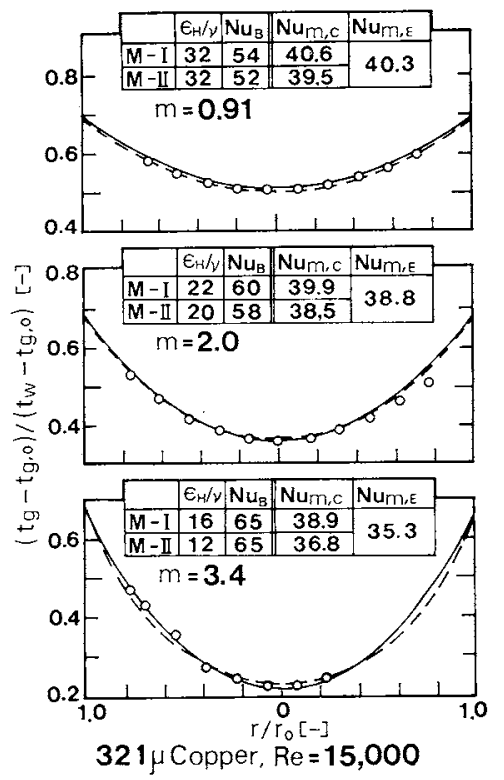

Fig. 4 Comparison of calculated gas temperature distributions with observed ones

Figure 5 compares the calculated overall Nusselt number ratio with the experimental one for the $1130 \mu$ glass beads as functions of loading ratio. The calculated ones are values evaluated by the parameters which give the best fit to the observed temperature distributions. There is a trend toward higher Nusselt number with increasing loading ratio in both calculated and observed values, after taking a minimum at lower 
loading ratios.

Gas eddy diffusivities of heat, giving the best fits to the experimental temperature distributions, are presented in Fig. 6 as functions of loading ratios. In the case of both 1130 and $181 \mu$ glass beads, gas eddy diffusivities decrease by addition of solids and take minima in the range of $m=2-4$, leading to almost constant values. These tendencies are similar for other conditions.

As for gas eddy diffusivity of heat in suspension flow, no experimental work has been reported. There are, however, two literature references furnishing us with much information about this problem. One is the work on the eddy diffusivity of heat in a pure gas flow within a pipe, where the value of $\varepsilon_{H} / \nu$ is within the range of $30-50$ at $R e=15,000^{11}$. The other is the measurement by Boothroyd ${ }^{2)}$ of gas eddy diffusivity of mass in a vertical suspension flow of zinc particles in the size range $0-40 \mu$. According to his result, the range of $\varepsilon_{c} / \nu$ from 40 to 70 in the case of $R e=$ 35,000 and a pipe diameter of 2 in., and lower values could be expected in the case of $R e=15,000$ and a pipe diameter of $1 \mathrm{in}$., which is the same condition as in our experiment. His eddy diffusivities show a minimum in the range $1<m<2$. He explained it as follows: The presence of the solids may be expected to attenuate the high-frequency types of turbulence characteristic of Newtonian fluids. Under suitable circumstances, however, where $\rho_{g} D^{2} / R e^{2}$ is sufficiently high, another form of turbulence field may develop in the sustenance of which the solid phase plays a greater part. The minimum value of $\varepsilon_{\sigma} / \nu$ may, therefore, be the net effect of attenuated single-phase type turbulence, while that which is characteristic of suspension flow is as yet undeveloped. Both effects would be expected to develop with increase of loading ratio and thus possibly account for the minimum observed. There is surely something in this explanation. But there is a great doubt that the decrease in overall Nusselt number would be caused by that variation of eddy diffusivity alone, because the overall Nusselt number decreases with increase in loading ratio when $\varepsilon_{H} / \nu$ is kept constant in the above models. This is, therefore, considered to be caused by the effects both of turbulence reduction of gas phase, leading to decrease in eddy diffusivity, and of deformation of gas temperature distribution due to poor sensitivity of the thermal boundary layer at the wall to the presence of solids, mentioned previously ${ }^{6}$.

The influence of solid concentration and eddy diffusivity distributions in the radial direction was investigated based on another model that introduces them into Model I. The results, not presented here, showed that their influence was scarcely responsible for the core flattening of gas temperature distributions which

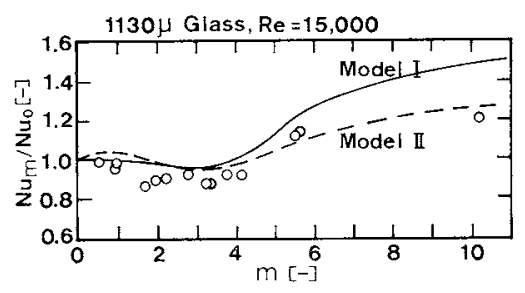

Fig 5 Comparison of calculated overall Nusselt numbers with observed ones

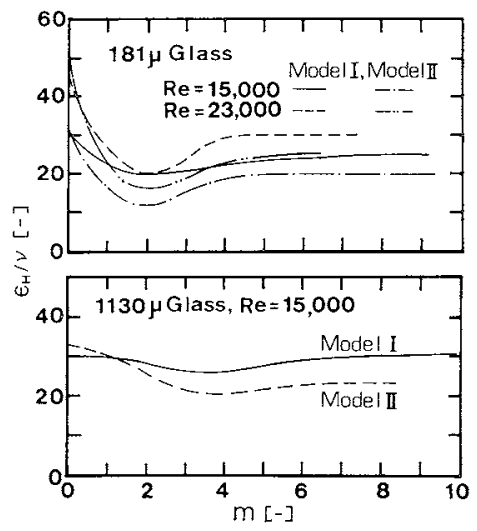

Fig. 6 Mean gas eddy diffusivity of heat as function of loading ratio

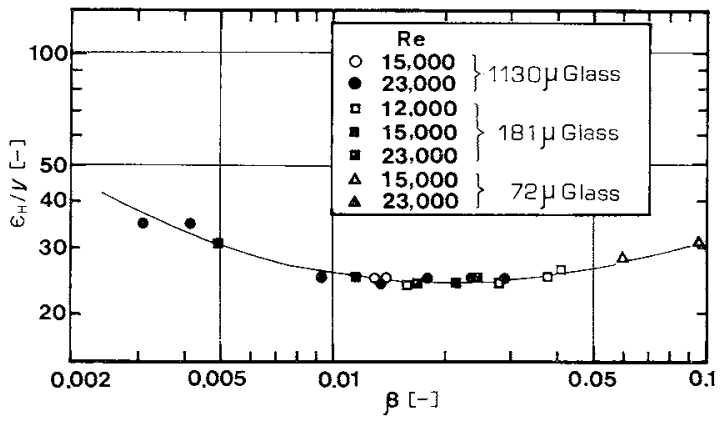

Fig. 7 Correlation of $\varepsilon_{H} / \nu$ with $\beta$

were not expressed well by Model I.

Finally, it would be surmised that the temperature distribution depends upon the radial eddy-mixing of solids. This can be conjectured from the fact that Model II expressed well a trend of temperature distribution toward flattening in the core region.

\section{2 Estimation of gas temperature distribution}

There are many cases in which dilute suspension flows can be seen in industrial processes, within the range of loading ratio lesser than unity. In those cases, temperature distributions of gas may be estimated well by using Model I. To apply Model I, however, we need values of two unknown parameters beforehand, even when the operating variables are given. Thus, we tried to correlate these parameters with the operating variables. The values of eddy diffusivity ratio $\varepsilon_{H} / \nu$ obtained are plotted against the nondimensional parameter $\beta$ in Fig. 7. It can be found from the figure that the $\varepsilon_{H} / \nu$ is correlated very well with $\beta$ less 


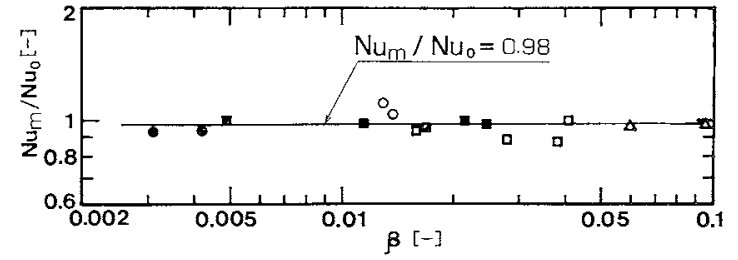

Fig. 8 Correlation of $N u_{m} / N u_{0}$ with $\beta$

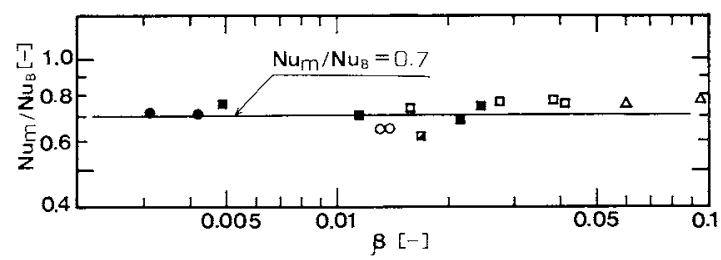

Fig. 9 Correlation of $N u_{m} / N u_{B}$ with $\beta$

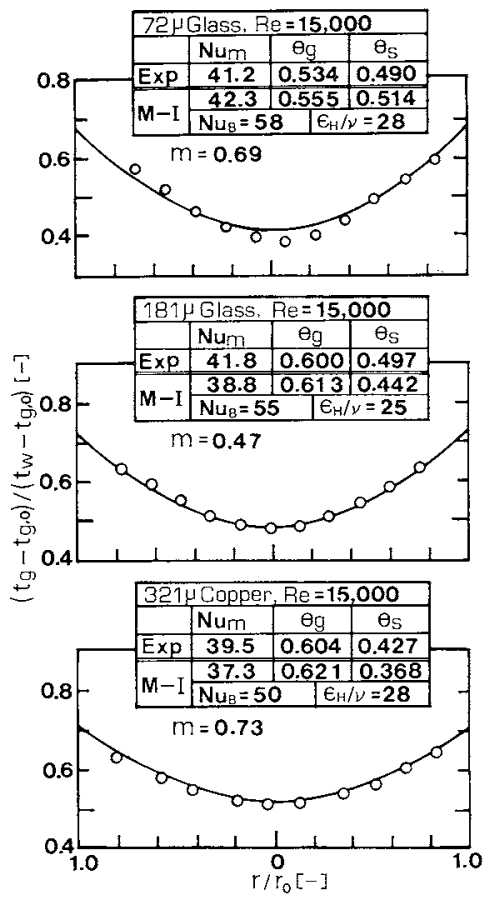

Fig. 10 Comparison of predicted gas temperature distributions with observed ones

than 0.1, for various particle sizes and Reynolds numbers. And for these cases, two kinds of Nusselt number ratios, $N u_{m} / N u_{0}$ and $N u_{m} / N u_{B}$, are also plotted against $\beta$ in Figs. 8 and 9. These figures show that both ratios are almost constant over the range covered, that is, $N u_{m} / N u_{0}=0.98$ and $N u_{m} / N u_{B}=0.7$, thus leading to $N u_{B} / N u_{0}=1.4$.

Consequently, temperature distributions of gas can be calculated based on Model I by using the two parameters evaluated through the above relations, if operating variables are given. In Fig. $\mathbf{1 0}$ are illustrated some examples of calculated distributions, compared with observed ones. $\theta_{s}$ in the figure is dimensionless mean solid temperature obtained by using a heat balance equation. Fairly good agreement can be seen in the overall Nusselt number as well as gas temperature distribution.

Finally, this study led to the conclusion that fairly accurate estimates of gas temperature distribution are obtained through the use of Model I in the range of loading ratio lower than about one.

\section{Conclusion}

Two mathematical models, quite different from each other in treatment of eddy-mixing of solid particles, were proposed to explain the heat transfer mechanism in gaseous suspension flow. Gas temperature distributions were expressed well by the models below a loading ratio of approximately 1.0. For higher loading ratios, however, they tended to flatten in the core region of pipe cross-section, and were not explained satisfactorily by these models. This core flattening may be closely related to solid eddy-mixing.

Mean gas eddy diffusivities of heat were obtained by these models through comparison of gas temperature distributions with observed ones, and correlated well with nondimensional parameter $\beta$ for lower loading ratios. This correlation can be used in the estimation of the gas temperature distributions on the basis of the above model.

\section{Appendix}

Let $\boldsymbol{J}_{0}[$ ] denote the finite Hankel transform operator, defined as

$$
\begin{aligned}
& J_{0}\left[T_{g}\right]=\int_{0}^{1} R T_{g} J_{0}\left(R \xi_{i}\right) d R=U_{i} \\
& J_{0}\left[T_{s}\right]=\int_{0}^{1} R T_{s} J_{0}\left(R \xi_{i}\right) d R=V_{i}
\end{aligned}
$$

where $J_{0}$ is Bessel function of first kind of order 0 and $\xi_{i}$ are positive roots of Eq. (12). Applying this transform, Eqs. (6)-(8) reduce to

$$
\begin{gathered}
d U_{i}=-\xi_{i}^{2} U_{i}-\beta\left(U_{i}-V_{i}\right) \\
d X \\
\frac{d V_{i}}{d X}=\gamma\left(U_{i}-V_{i}\right) \\
\text { at } X=0 \quad U_{i}=J_{0}[1]=J_{1}\left(\xi_{i}\right) / \xi_{i}=U_{0} \\
V_{i}=J_{0}\left[T_{s, 0}\right]=T_{s, 0} J_{1}\left(\hat{\xi}_{i}\right) / \xi_{i}=V_{0}
\end{gathered}
$$

Solutions of these equations can be obtained easily, and thus functions $T_{g}$ and $T_{s}$ are given by the following inverse formula ${ }^{7}$ :

$$
\begin{aligned}
& T_{g}=2 \sum_{i=1}^{\infty} \frac{\xi_{i}{ }^{2} U_{i}}{\left(B^{2}+\xi_{i}{ }^{2}\right)} \frac{J_{0}\left(R \xi_{i}\right)}{\left[J_{0}\left(\xi_{i}\right)\right]^{2}} \\
& T_{s}=2 \sum_{i=1}^{\infty} \frac{\xi_{i}{ }^{2} V_{i}}{\left(B^{2}+\xi_{i}{ }^{2}\right)} \frac{J_{0}\left(R \xi_{i}\right)}{\left[J_{0}\left(\xi_{i}\right)\right]^{2}}
\end{aligned}
$$

\section{Nomenclature}

$A_{p} \quad=$ surface area of a single particle $\quad\left[\mathrm{m}^{2}\right]$

$a_{1} \quad=$ auxiliary variable $\quad[-]$

$B \quad=h_{B} r_{0} / \rho_{g} C_{p,{ }} \varepsilon_{H}=N u_{B} / 2 P r \cdot\left(\varepsilon_{H} / \nu\right) \quad[-]$

$b_{1} \quad=$ auxiliary variable $\quad[-]$

$C_{p}=$ heat capacity at constant pressure $\left[\mathrm{Kcal} / \mathrm{kg} \cdot{ }^{\circ} \mathrm{C}\right]$

$c_{1}, c_{2} \quad=$ auxiliary variables $[-]$

$D \quad=$ inside tube diameter [m]

$\begin{array}{lll}d_{p} & =\text { particle diameter } & \text { [m] }\end{array}$ 


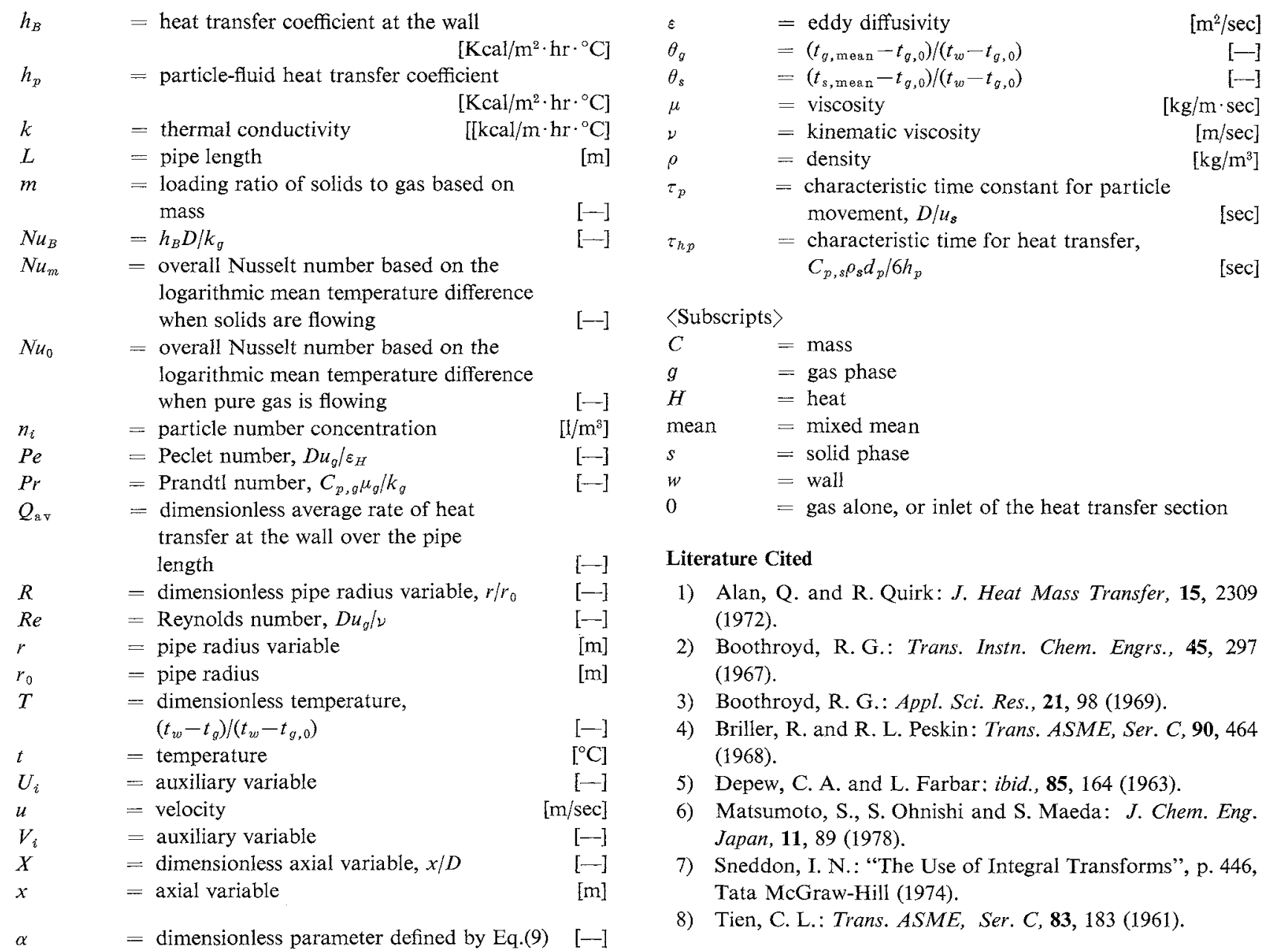

(A part of this paper was presented at Tohoku Meeting of The Soc. of Chem. Engrs., Japan, at Yonezawa, July, 1976.) 